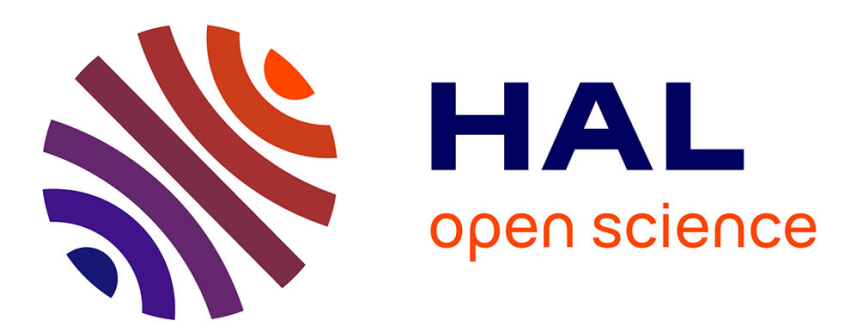

\title{
Homogenization of the three-dimensional Hall effect and change of sign of the Hall coefficient
}

\author{
Marc Briane, Graeme W. Milton
}

\section{To cite this version:}

Marc Briane, Graeme W. Milton. Homogenization of the three-dimensional Hall effect and change of sign of the Hall coefficient. Archive for Rational Mechanics and Analysis, 2009, 193 (3), pp.715-738. 10.1007/s00205-008-0200-y . hal-00130591v2

\section{HAL Id: hal-00130591 \\ https://hal.science/hal-00130591v2}

Submitted on 20 Jul 2007

HAL is a multi-disciplinary open access archive for the deposit and dissemination of scientific research documents, whether they are published or not. The documents may come from teaching and research institutions in France or abroad, or from public or private research centers.
L'archive ouverte pluridisciplinaire HAL, est destinée au dépôt et à la diffusion de documents scientifiques de niveau recherche, publiés ou non, émanant des établissements d'enseignement et de recherche français ou étrangers, des laboratoires publics ou privés. 


\title{
Homogenization of the three-dimensional Hall effect and change of sign of the Hall coefficient
}

\author{
Marc BRIANE \\ Centre de Math., I.N.S.A. de Rennes \& I.R.M.A.R. \\ 35043 Rennes Cedex, FRANCE \\ mbriane@insa-rennes.fr
}

\author{
Graeme W. MILTON \\ Dept. of Math., University of Utah \\ Salt Lake City, Utah 84112-0090, USA \\ milton@math.utah.edu
}

July 20, 2007

\begin{abstract}
The notion of a Hall matrix, associated with a possibly anisotropic conducting material in the presence of a small magnetic field, is introduced. Then, for any material having a microstructure we prove a general homogenization result satisfied by the Hall matrix in the framework of the $H$ convergence of Murat-Tartar. Extending a result of Bergman, it is shown that the Hall matrix can be computed from the corrector associated with the homogenization problem when no magnetic field is present. Finally, we give an example of a microstructure for which the Hall matrix is positive isotropic a.e., while the homogenized Hall matrix is negative isotropic.
\end{abstract}

\section{Introduction}

It is well known that a charged particle moving perpendicular to a magnetic field $h$ will experience a force perpendicular to both the direction of motion of the particle and to the magnetic field. When applied to a charge carrier moving in a conductor, the classical explanation of the Hall effect is that this force has to be balanced by a transverse electric field, and in an isotropic material, the ratio between the transverse component of the electric field and the current defines the Hall coefficient. This simple picture underlies the statement found in some elementary Physics textbooks (see e.g. [12] page 163), that in classical physics the sign of the Hall coefficient determines the sign of the charge carrier. But is this really correct, even within the framework of classical physics? If it were, it would suggest that an electrically isotropic composite constructed from materials with positive Hall coefficients for which the carriers had a negative charge, would necessarily also have a positive effective Hall coefficient. But we will see that this is not always the case. The situation is a little reminiscent to that of thermoelasticity where it was found by Lakes [8] and Sigmund and Torquato [13, 14] that one can construct a composite that contracts when heated, although the three constituent materials all expand when heated. (The void phase in these constructions can be replaced by a highly compressible phase with a very small positive thermal expansion coefficient.)

Before presenting the example of an electrically isotropic composite having a negative Hall coefficient even though it is built from three phases each having a positive Hall coefficient (equal to 1 or $\gamma \ll 1$ ), we first place the theory of the Hall effect in composites in a general mathematical framework. For anisotropic materials we will see that it is natural to introduce the concept of a Hall matrix. An extension of the analysis of Bergman [3] shows that for composites this matrix can be obtained from the electric fields (more precisely the correctors) associated with the material when the magnetic field is absent.

We consider a small constant magnetic field $h$ in a three-dimensional microstructure which occupies a bounded domain $\Omega$ of $\mathbb{R}^{3}$, and which is characterized by a $h$-dependent conductivity $\sigma^{\varepsilon}$ or resistivity $\rho^{\varepsilon}=\left(\sigma^{\varepsilon}\right)^{-1}$. Here, as usual in homogenization theory, $\varepsilon$ labels one microstructure in a general sequence 
of microstructures. Mathematically speaking the Hall effect induces a perturbed resistivity $\rho^{\varepsilon}(h)$, with parameter $h$, which admits the first-order expansion at $h=0$ :

$$
\rho^{\varepsilon}(h)=\rho^{\varepsilon}+\mathcal{R}_{1}^{\varepsilon} \cdot h+o(h),
$$

where $\mathcal{R}_{1}^{\varepsilon}$ is a third-order tensor. The first-order term of expansion (1.1), which is necessarily an antisymmetric matrix, contains the local Hall effect. At this point we address the following question: What is the effective or the homogenized Hall effect of the composite material, i.e. associated with the homogenized microstructure?

Assuming a periodic microstructure, Bergman [3] first answered that the effective Hall coefficient induced by a constant magnetic field parallel to some vector $e_{3}\left(\left(e_{1}, e_{2}, e_{3}\right)\right.$ being an orthonormal basis of $\mathbb{R}^{3}$ ), can be obtained from the local periodic Hall coefficient combined with the two periodic current fields of average $e_{1}, e_{2}$ orthogonal to $e_{3}$, which solve the conductivity equation in the absence of magnetic field. In dimension two the Bergman approach was rigorously justified and extended in the paper [4] in the $H$-convergence framework of Murat-Tartar [11], which is not restricted to the periodic case.

The two-dimensional case leads us naturally to a unique Hall coefficient since any antisymmetric matrix is proportional to the $90^{\circ}$ rotation matrix $J$. Then, the first-order term of the expansion of $\rho^{\varepsilon}(h)$ reads as $r_{\varepsilon} h J$ while the corresponding homogenized one reads as $r_{*} h J$. So, $r_{\varepsilon}$ is the Hall coefficient associated with the microstructure and $r_{*}$ is the effective Hall coefficient. A remarkable property of the two-dimensional Hall effect is that $r_{\varepsilon}$ does keep the sign of $r_{*}$, i.e. $r_{\varepsilon} \geq 0$ a.e. in $\Omega$ implies that $r_{*} \geq 0$ a.e. in $\Omega$.

Defining in a general way the three-dimensional Hall coefficients for any constant magnetic field is more delicate than in dimension two. In this paper we extend the Bergman approach by introducing the concept of a Hall matrix. Using the Levi-Civita third-order tensor $\mathcal{E}$ (see Definition 3.1), the antisymmetric first-order term of expansion (1.1) can be expressed as $\mathcal{E}\left(R^{\varepsilon} h\right)$ for a certain choice of the matrix $R^{\varepsilon}$ and the homogenized can be expressed as $\mathcal{E}\left(R^{*} h\right)$ for a certain choice of the matrix $R^{*}$. So, it is natural to define $R^{\varepsilon}$ as the Hall matrix and $R^{*}$ as the homogenized one. One appealing feature of this definition is that if $R^{\varepsilon}$ does not depend on $\varepsilon$, then $R^{\varepsilon}$ and $R^{*}$ agree (see Proposition 4.1).

Moreover, extending the above mentioned Bergman periodic formula for the effective Hall coefficients, we prove (see Theorem 3.6) that the effective Hall matrix $R^{*}$ is obtained from the Hall matrix $R^{\varepsilon}$ combined with the current field $\sigma^{\varepsilon} P^{\varepsilon}$, where $P^{\varepsilon}$ is the corrector associated with $\sigma^{\varepsilon}$, i.e. the electric field weakly converging to $I$ and solving the conductivity equation in the absence of a magnetic field (see Definition 2.4).

We then show that in contrast to the two-dimensional Hall effect studied in [4], the homogenization process from $R^{\varepsilon}$ to $R^{*}$ in three dimensions does not preserve any positivity property. Indeed, we give (see Theorem 4.2) an example of a microstructure for which $R^{\varepsilon}$ is positive isotropic a.e., while $R^{*}$ is a constant negative isotropic matrix. This surprising result is linked to the change of sign of the corrector's determinant derived in [5].

The paper is organized as follows. In Section 2 we recall a few results of $H$-convergence which provides a general framework for studying the Hall effect. In Section 3 we introduce the notion of the three-dimensional Hall matrix associated with a microstructure and we prove a homogenization result about the Hall matrix. Section 4 is devoted to the example where the composite is electrically isotropic, but the effective Hall coefficient has opposite sign to that of the constituents.

\section{Notations}

- $Y$ denotes the cube $(-1,1)^{d}, d \geq 2$, and $\Omega$ a bounded open set of $\mathbb{R}^{d}$;

- $I$ denotes the unit matrix of $\mathbb{R}^{d \times d}$;

- for any $A \in \mathbb{R}^{d \times d}, A^{T}$ denotes the transpose of $A, \operatorname{det}(A)$ its determinant, $\operatorname{tr}(A)$ its trace, and $\operatorname{Cof}(A)$ its cofactor matrix; 
- $|\cdot|$ denotes the euclidian norm in $\mathbb{R}^{d}$ and the subordinate norm in $\mathbb{R}^{d \times d}$, i.e., for any $A \in \mathbb{R}^{d \times d}$, $|A|:=\sup \{|A x|:|x|=1\}$

- $\mathbb{R}_{a}^{d \times d}$ denotes the set of the antisymmetric matrices in $\mathbb{R}^{d \times d}$;

- for any $\alpha, \beta>0, \mathcal{M}(\alpha, \beta ; \Omega)$ is the set of the matrix-valued functions $A: \Omega \longrightarrow \mathbb{R}^{d \times d}$ such that

$$
\forall \xi \in \mathbb{R}^{d}, \quad A(x) \xi \cdot \xi \geq \alpha|\xi|^{2} \quad \text { and } \quad A^{-1}(x) \xi \cdot \xi \geq \beta^{-1}|\xi|^{2}, \quad \text { a.e. } x \in \Omega ;
$$

- $H_{\#}^{1}(Y)$ denotes the space of functions which are $Y$-periodic in $\mathbb{R}^{d}$ and belong to $H_{\text {loc }}^{1}\left(\mathbb{R}^{d}\right)$;

- for any $u: \mathbb{R}^{d} \longrightarrow \mathbb{R}, \nabla u:=\left(\frac{\partial u}{\partial x_{i}}\right)_{1 \leq i \leq d}$;

- for any $U: \mathbb{R}^{d} \longrightarrow \mathbb{R}^{d}, U=\left(u_{1}, u_{2}, u_{3}\right), D U:=\left(\frac{\partial u_{j}}{\partial x_{i}}\right)_{1 \leq i, j \leq d}$;

- for any $\sigma: \mathbb{R}^{d} \longrightarrow \mathbb{R}^{d \times d}, \operatorname{Div}(\sigma):=\left(\frac{\partial \sigma_{i j}}{\partial x_{i}}\right)_{1 \leq j \leq d}$ and $\operatorname{Curl}(\sigma):=\left(\frac{\partial \sigma_{i k}}{\partial x_{j}}-\frac{\partial \sigma_{j k}}{\partial x_{i}}\right)_{1 \leq i, j, k \leq d}$;

- for any sequence of functions $f_{\varepsilon}: O \longrightarrow H, \varepsilon>0$, where $O$ is a neighbourhood of 0 in $\mathbb{R}^{d}$ and $(H,\|\cdot\|)$ a Banach space, we use the following convention

$$
f_{\varepsilon}(h)=o_{H}(h) \Longleftrightarrow \lim _{h \rightarrow 0}\left(\frac{1}{|h|} \sup _{\varepsilon>0}\left\|f_{\varepsilon}(h)\right\|\right)=0
$$

i.e. the $o_{H}(h)$ is uniform with respect to $\varepsilon$;

- $\mathcal{D}^{\prime}(\Omega)$ denotes the space of the distributions on $\Omega$.

\section{Review of homogenization}

\section{$2.1 H$-convergence}

Let $\Omega$ be a bounded open set of $\mathbb{R}^{d}$ and let $\alpha, \beta>0$. We recall here the definition of $H$-convergence due to Murat and Tartar [11] of a sequence of matrix-valued functions in $\mathcal{M}(\alpha, \beta ; \Omega)$ (see notation $(1.2)$ ), some properties of the $H$-convergence, and the definition of a corrector:

Definition 2.1. (Murat-Tartar) A sequence $A^{\varepsilon}$ in $\mathcal{M}(\alpha, \beta ; \Omega)$ is said to $H$-converge to $A^{*}$ in $\mathcal{M}(\alpha, \beta ; \Omega)$ if any sequence $u_{\varepsilon}$ in $H_{0}^{1}(\Omega)$ such that $\operatorname{div}\left(A^{\varepsilon} \nabla u_{\varepsilon}\right)$ strongly converges to $f$ in $H^{-1}(\Omega)$ as $\varepsilon \rightarrow 0$, weakly converges in $H_{0}^{1}(\Omega)$ to the solution $u$ of $\operatorname{div}(A \nabla u)=f$, and the sequence $A^{\varepsilon} \nabla u_{\varepsilon}$ weakly converges to $A^{*} \nabla u$ in $L^{2}(\Omega)^{d}$. Then, the matrix-valued function $A^{*}$ is called the homogenized matrix or the $H$-limit of $A^{\varepsilon}$.

Theorem 2.2. (Murat-Tartar) Any sequence in $\mathcal{M}(\alpha, \beta ; \Omega)$ admits a subsequence which $H$-converges to a matrix-valued function in $\mathcal{M}(\alpha, \beta ; \Omega)$.

Proposition 2.3. (Murat-Tartar) If $A^{\varepsilon}$ is a sequence in $\mathcal{M}(\alpha, \beta ; \Omega)$ which $H$-converges to $A^{*}$, then $\left(A^{\varepsilon}\right)^{T} H$-converges to $\left(A^{*}\right)^{T}$.

Definition 2.4. (Murat-Tartar) Let $A^{\varepsilon}$ be a sequence in $\mathcal{M}(\alpha, \beta ; \Omega)$ which $H$-converges to $A^{*}$. Any matrix-valued function $P^{\varepsilon}$ in $L^{2}(\Omega)^{3}$ satisfying

$$
\left\{\begin{array}{rll}
P^{\varepsilon} & \longrightarrow I & \text { weakly in } L^{2}(\Omega)^{d \times d} \\
\operatorname{Curl}\left(P^{\varepsilon}\right) & \longrightarrow 0 & \text { strongly in } H^{-1}(\Omega)^{d \times d \times d} \\
\operatorname{Div}\left(A^{\varepsilon} P^{\varepsilon}\right) & \longrightarrow \operatorname{Div}\left(A^{*}\right) & \text { strongly in } H^{-1}(\Omega)^{d \times d}
\end{array}\right.
$$


is called a corrector associated with $A^{\varepsilon}$. In this case, the following convergence also holds true

$$
A^{\varepsilon} P^{\varepsilon} \longrightarrow A^{*} \quad \text { weakly in } L^{2}(\Omega)^{d \times d} \text {. }
$$

The following result allows us to build correctors:

Proposition 2.5. Let $A^{\varepsilon}$ be a sequence in $\mathcal{M}(\alpha, \beta ; \Omega)$ which $H$-converges to $A^{*}$. Let $U^{\varepsilon}$ be the solution in $H^{1}(\Omega)^{3}$ of the equation

$$
\begin{cases}\operatorname{Div}\left(A^{\varepsilon} D U^{\varepsilon}\right)=\operatorname{Div}\left(A^{*}\right) & \text { in } \Omega \\ U^{\varepsilon}(x)=x & \text { on } \partial \Omega .\end{cases}
$$

Then, $D U^{\varepsilon}$ is a corrector associated with $A^{\varepsilon}$ and, for any corrector $P^{\varepsilon}$ associated with $A^{\varepsilon}$, the sequence $P^{\varepsilon}-D U^{\varepsilon}$ strongly converges to 0 in $L_{\mathrm{loc}}^{2}(\Omega)^{3 \times 3}$.

Example 2.6. Let $A$ be a $Y$-periodic matrix-valued function in $\mathcal{M}\left(\alpha, \beta ; \mathbb{R}^{d}\right)$ and define, for any $\varepsilon>0$, $A^{\varepsilon}(x):=A\left(\frac{x}{\varepsilon}\right)$ a.e. $x \in \Omega$. Then, the sequence $A^{\varepsilon} H$-converges to a constant matrix. Moreover, if $U$ is the unique solution, with zero $Y$-average, of equation

$$
\left\{\begin{aligned}
\operatorname{Div}(A D U)=0 & \text { in } \mathcal{D}^{\prime}\left(\mathbb{R}^{d}\right) \\
U(y)-y & \in H_{\#}^{1}(Y)^{d},
\end{aligned}\right.
$$

then the matrix-valued function defined by $P^{\varepsilon}(x):=D U\left(\frac{x}{\varepsilon}\right)$ is a corrector associated with $A^{\varepsilon}$.

\section{$2.2 \quad H$-convergence with a parameter}

We now consider a sequence $A^{\varepsilon}(h)$ in $\mathcal{M}(\alpha, \beta ; \Omega)$, with a parameter $h$ belonging to a neighbourhood of 0 in $\mathbb{R}^{d}$. We have the following differentiability result satisfied by the homogenized matrix $A^{*}(h)$ at $h=0$ :

Theorem 2.7. ( $i)$ Assume that $A^{\varepsilon}(h)$ in $\mathcal{M}(\alpha, \beta ; \Omega)$ satisfies the uniform Lipschitz condition

$$
\exists C>0, \forall h, h^{\prime} \in O, \quad\left|A^{\varepsilon}(h)-A^{\varepsilon}\left(h^{\prime}\right)\right| \leq C\left|h-h^{\prime}\right|,
$$

and the differentiability at $h=0$ (see notation (1.3))

$$
A^{\varepsilon}(h)=A^{\varepsilon}+\mathcal{A}_{1}^{\varepsilon} \cdot h+o_{L^{\infty}(\Omega)^{d \times d}}(h),
$$

where $A^{\varepsilon}:=A^{\varepsilon}(0)$ and $\mathcal{A}_{1}^{\varepsilon}$ is a bounded sequence in $L^{\infty}(\Omega)^{d \times d \times d}$. Then, there exists a subsequence, still denoted by $\varepsilon$, such that, for any $h \in O, A^{\varepsilon}(h) H$-converges to some $A^{*}(h)$ in $\mathcal{M}(\alpha, \beta ; \Omega)$. Moreover, the $H$-limit $A^{*}$ satisfies the first-order expansion at $h=0$

$$
A^{*}(h)=A^{*}+\mathcal{A}_{1}^{*} \cdot h+o_{L^{2}(\Omega) d \times d}(h),
$$

where $A^{*}:=A^{*}(0)$ and $\mathcal{A}_{1}^{*} \in L^{2}(\Omega)^{d \times d \times d}$.

(ii) Also assume that the matrix $A^{\varepsilon}$ of (2.6) is symmetric and let $P^{\varepsilon}$ be a corrector (2.1) associated with $A^{\varepsilon}$. Then, the following convergence holds true in the sense of distributions:

$$
\forall h \in O, \quad\left(P^{\varepsilon}\right)^{T}\left(\mathcal{A}_{1}^{\varepsilon} \cdot h\right) P^{\varepsilon} \longrightarrow \mathcal{A}_{1}^{*} \cdot h \quad \text { in } \mathcal{D}^{\prime}(\Omega)^{d \times d} .
$$

Remark 2.8. Part $(i)$ of Theorem 2.7 is proved in [4]. It is an extension of Theorem 2.5 in [6] where $A^{\varepsilon}(h)$ is of class $C^{n}$ with respect to $h$ and its $n+1$ derivatives satisfy the Lipschitz condition (2.5). Under this stronger assumption of regularity the $H$-limit is also of class $C^{n}$ and its derivatives are uniformly Lipschitz. Here, we only assume the differentiability (2.6) at $h=0$ combined with (2.5). In return, the first-order expansion $(2.7)$ of $A^{*}(h)$ is only valid in the sense of the $L^{2}(\Omega)^{d \times d}$-norm.

Part (ii) of Theorem 2.7 is proved in the appendix. 


\section{The Hall effect and homogenization}

\subsection{The three-dimensional Hall effect}

The dimension is $d=3$ from now onwards. Let $\Omega$ be a bounded open subset of $\mathbb{R}^{3}$, let $O$ be a ball of $\mathbb{R}^{3}$ centered at 0 , and let $\alpha, \beta>0$. Consider a sequence $\sigma^{\varepsilon}(h)$, for $\varepsilon>0$ and $h \in O$, of matrix-valued functions in $\mathcal{M}(\alpha, \beta ; \Omega)$, which represents the conductivity matrix of a heterogeneous conducting material in the presence of a constant magnetic field $h$, and the microstructure of which is measured by the small parameter $\varepsilon$. From the physics of the problem it can be shown (see e.g. Section 21 of [9] pages $132-135)$ that $\sigma^{\varepsilon}(h)$ satisfies the property

$$
\forall h \in \mathbb{R}^{3}, \quad \sigma^{\varepsilon}(h)^{T}=\sigma^{\varepsilon}(-h),
$$

which implies that the symmetric part of $\sigma^{\varepsilon}(h)$ is even and the antisymmetric one is odd with respect to $h$. Then, in the presence of a low magnetic field $h$ we can assume that the following first-order expansion holds at $h=0$ :

$$
\sigma^{\varepsilon}(h)=\sigma^{\varepsilon}+\mathcal{S}_{1}^{\varepsilon} \cdot h+o_{L^{\infty}(\Omega)^{3 \times 3}}(h),
$$

where $\sigma^{\varepsilon}=\sigma^{\varepsilon}(0)$ and $\mathcal{S}_{1}^{\varepsilon}$ is a bounded sequence in $L^{\infty}(\Omega)^{3 \times 3 \times 3}$. We also assume that $\sigma^{\varepsilon}(h)$ satisfies the uniform Lipschitz condition (2.5) in $O$. Therefore, it is easy to check that the resistivity $\rho^{\varepsilon}(h):=$ $\sigma^{\varepsilon}(h)^{-1}$ belongs to $\mathcal{M}\left(\beta^{-1}, \alpha^{-1} ; \Omega\right)$, and also satisfies the uniform Lipschitz condition (2.5) in $O$, as well as the first-order expansion

$$
\rho^{\varepsilon}(h)=\rho^{\varepsilon}+\mathcal{R}_{1}^{\varepsilon} \cdot h+o_{L^{\infty}(\Omega)^{3 \times 3}}(h),
$$

where $\rho^{\varepsilon}:=\rho^{\varepsilon}(0)$ and $\mathcal{R}_{1}^{\varepsilon}$ is a bounded sequence in $L^{\infty}(\Omega)^{3 \times 3 \times 3}$. The first-order term of expansion (3.3) corresponds to the Hall effect due to the magnetic field $h$ which perturbs the resistivity $\rho^{\varepsilon}$.

By virtue of property (3.1) the matrix-valued functions $\sigma^{\varepsilon}$ and $\rho^{\varepsilon}$ are symmetric while $\mathcal{S}_{1}^{\varepsilon} \cdot h$ and $\mathcal{R}_{1}^{\varepsilon} \cdot h$ are antisymmetric for any $h \in O$. Therefore, the third-order tensors $\mathcal{S}_{1}^{\varepsilon}$ and $\mathcal{R}_{1}^{\varepsilon}$ define two linear mappings from $\mathbb{R}^{3}$ into the space $\mathbb{R}_{a}^{3 \times 3}$ of antisymmetric matrices in $\mathbb{R}^{3 \times 3}$. This leads us to the following definition of the Hall matrix:

Definition 3.1. Let $\mathcal{E}$ be the Levi-Civita third-order tensor (see e.g. [17]) defined by the one-to-one linear mapping

$$
\begin{aligned}
& \mathcal{E}: \mathbb{R}^{3} \longrightarrow \mathbb{R}_{a}^{3 \times 3} \\
& \xi \longmapsto \mathcal{E}(\xi):=\left[e_{i j k} \xi_{k}\right]_{1 \leq i, j \leq 3},
\end{aligned}
$$

with the Einstein convention of summing over the repeated subscript, and where

$$
e_{i j k}:=\left\{\begin{array}{cl}
1 & \text { if }(i, j, k) \text { is an even permutation of }(1,2,3) \\
-1 & \text { if }(i, j, k) \text { is an odd permutation of }(1,2,3) \\
0 & \text { otherwise }
\end{array}\right.
$$

Then, the Hall matrix $R^{\varepsilon}$ associated with the resistivity $\rho^{\varepsilon}(h)$ is defined from the first-order term of (3.3) by

$$
R^{\varepsilon} h:=\mathcal{E}^{-1}\left(\mathcal{R}_{1}^{\varepsilon} \cdot h\right), \quad \text { for any } h \in O,
$$

to ensure that

$$
\rho^{\varepsilon}(h)=\rho^{\varepsilon}+\mathcal{E}\left(R^{\varepsilon} h\right)+o_{L^{\infty}(\Omega)^{3 \times 3}}(h) .
$$

Notice that the action of $\mathcal{E}^{-1}$ is easy to compute, since in coordinates

$$
\mathcal{E}^{-1}\left(\begin{array}{ccc}
0 & a_{1} & a_{2} \\
-a_{1} & 0 & a_{3} \\
-a_{2} & -a_{3} & 0
\end{array}\right)=\left(\begin{array}{c}
a_{3} \\
-a_{2} \\
a_{1}
\end{array}\right) \quad \text { for } a_{1}, a_{2}, a_{3} \in \mathbb{R} .
$$


Remark 3.2. Since by assumption $\mathcal{R}_{1}^{\varepsilon}$ is a bounded sequence in $L^{\infty}(\Omega)^{3 \times 3 \times 3}, R^{\varepsilon}$ is a bounded sequence of matrix-valued functions in $L^{\infty}(\Omega)^{3 \times 3}$. Similar to (3.7) there exists a bounded sequence $S^{\varepsilon}$ in $L^{\infty}(\Omega)^{3 \times 3}$ such that the first-order expansion (3.2) reads as

$$
\sigma^{\varepsilon}(h)=\sigma^{\varepsilon}+\mathcal{E}\left(S^{\varepsilon} h\right)+o_{L^{\infty}}(\Omega)^{3 \times 3}(h) .
$$

Remark 3.3. In dimension two the Hall matrix is replaced by a scalar coefficient (see [4]).

\subsection{Homogenization of the Hall effect}

On the one hand, due to the Lipschitz condition (2.5) and the compactness of the $H$-convergence in Theorem 2.2, there exists a subsequence of $\varepsilon$, still denoted by $\varepsilon$, such that the conductivity $\sigma^{\varepsilon}(h)$ $H$-converges to some $\sigma^{*}(h)$ in $\mathcal{M}(\alpha, \beta ; \Omega)$, for any $h \in O$. Since $\sigma^{\varepsilon}(h)^{T}=\sigma^{\varepsilon}(-h) H$-converges to $\sigma^{*}(h)^{T}$ by Proposition 2.3, we also get $\sigma^{*}(h)^{T}=\sigma^{*}(-h)$ for any $h \in O$. Therefore, by the firstorder expansion (2.7) of Theorem 2.7 and similar to (3.2), there exists a matrix-valued $S^{*}$ in $L^{2}(\Omega)^{3 \times 3}$ such that

$$
\sigma^{*}(h)=\sigma^{*}+\mathcal{E}\left(S^{*} h\right)+o_{L^{2}(\Omega)^{3 \times 3}}(h),
$$

where $\sigma^{*}=\sigma^{*}(0) \in \mathcal{M}(\alpha, \beta ; \Omega)$.

On the other hand, taking the inverse of expansion (3.10) the effective resistivity $\rho^{*}(h):=\left[\sigma^{*}(h)\right]^{-1}$ satisfies the similar expansion

$$
\rho^{*}(h)=\rho^{*}+\mathcal{E}\left(R^{*} h\right)+o_{L^{2}(\Omega)^{3 \times 3}(h),}
$$

where $\rho^{*}=\rho^{*}(0) \in \mathcal{M}\left(\beta^{-1}, \alpha^{-1} ; \Omega\right)$. This leads us to the following definition:

Definition 3.4. The matrix-valued $R^{*}$ defined by the first-order expansion (3.11) is the effective Hall matrix of $R^{\varepsilon}$ defined by (3.7).

To distinguish the Hall matrices $R^{\varepsilon}, R^{*}$ from the matrices $S^{\varepsilon}, S^{*}$, the last ones will be called the $S$-matrices in what follows. Similarly, with regard to the Hall coefficients $r_{\varepsilon}, r_{*}$, the coefficients $s_{\varepsilon}, s_{*}$ will be called the $s$-coefficients.

Proposition 3.5. The Hall matrices $R^{\varepsilon}, R^{*}$ and the $S$-matrices $S^{\varepsilon}, S^{*}$ are linked by the following relations

$$
S^{\varepsilon}=-\operatorname{Cof}\left(\sigma^{\varepsilon}\right) R^{\varepsilon} \quad \text { and } \quad S^{*}=-\operatorname{Cof}\left(\sigma^{*}\right) R^{*} .
$$

The homogenization problem is now to derive the pair $\left(\sigma^{*}, S^{*}\right)$ (or equivalently the pair $\left(\rho^{*}, R^{*}\right)$ ) of homogenized matrices from the sequence $\left(\sigma^{\varepsilon}, S^{\varepsilon}\right)$ (or from $\left(\rho^{\varepsilon}, R^{\varepsilon}\right)$ ). The result for $S^{*}$ (or for the effective Hall matrix $R^{*}$ ) simply depends on the solutions for the fields (electric or current) without any magnetic field present as shown by the following result:

Theorem 3.6. Let $P^{\varepsilon}$ be a corrector associated with the conductivity sequence $\sigma^{\varepsilon}$ according to Definition 2.4. Then, the homogenized $S$-matrix $S^{*}$ (3.10) is derived from the sequence $S^{\varepsilon}$ by the following convergence in the distribution sense

$$
\operatorname{Cof}\left(P^{\varepsilon}\right)^{T} S^{\varepsilon} \longrightarrow S^{*} \text { in } \mathcal{D}^{\prime}(\Omega)^{3 \times 3}
$$

while the effective Hall matrix $R^{*}$ (3.11) is given by

$$
\operatorname{Cof}\left(\sigma^{\varepsilon} P^{\varepsilon}\right)^{T} R^{\varepsilon} \longrightarrow \operatorname{Cof}\left(\sigma^{*}\right)^{T} R^{*}=\operatorname{Cof}\left(\sigma^{*}\right) R^{*} \text { in } \mathcal{D}^{\prime}(\Omega)^{3 \times 3} .
$$

Remark 3.7. The electric field $P^{\varepsilon}$ arises in the homogenization process (3.13) giving the $S$-matrix $S^{*}$ associated with the conductivity $\sigma^{*}$ by (3.10). However, it is the current field $\sigma^{\varepsilon} P^{\varepsilon}$ which arises in the limit process (3.14) giving the effective Hall matrix $R^{*}$. 


\subsection{Proof of the results}

The proof of Theorem 3.6 is based of the following algebraic result:

Lemma 3.8. For any vector $\xi$ in $\mathbb{R}^{3}$ and any symmetric matrix $P$ in $\mathbb{R}^{3 \times 3}$, the Levi-Civita tensor $\mathcal{E}$ defined by (3.4) satisfies the relation

$$
P^{T} \varepsilon(\xi) P=\mathcal{E}\left(\operatorname{Cof}(P)^{T} \xi\right)
$$

Proof. Let $\xi \in \mathbb{R}^{3}$, let $P$ be an invertible matrix in $\mathbb{R}^{3 \times 3}$, and set $\eta:=P^{-1} \xi$. Let $i, j \in\{1,2,3\}$, we have using Einstein's convention

$$
\left[P^{T} \mathcal{E}(P \eta) P\right]_{i j}=\left(P^{T}\right)_{i k}[\mathcal{E}(P \eta)]_{k l} P_{l j}=P_{k i} e_{k l m}[P \eta]_{m} P_{l j}=e_{k l m} P_{k i} P_{l j} P_{m n} \eta_{n}
$$

Fix $i, j, n \in\{1,2,3\}$. If two integers among $i, j, n$ are equal, for example $i=j$, we have

$$
e_{k l m} P_{k i} P_{l j} P_{m n}=e_{k l m} P_{l i} P_{k i} P_{m n}=-e_{l k m} P_{l i} P_{k i} P_{m n}=-e_{k l m} P_{k i} P_{l j} P_{m n}
$$

hence $e_{k l m} P_{k i} P_{l j} P_{m n}=0=\operatorname{det}(P) e_{i j n}$. Now, assume that $i, j, n$ are distinct integers. If $(i, j, n)$ is an even permutation of $(1,2,3)$, since $e_{k l m}$ is invariant by an even permutation of $(k, l, m)$, we have

$$
e_{k l m} P_{k i} P_{l j} P_{m n}=e_{k l m} P_{k 1} P_{l 2} P_{m 3}=\operatorname{det}(P)=\operatorname{det}(P) e_{i j n} .
$$

Otherwise, since $e_{k l m}$ changes sign by an odd permutation of $(k, l, m)$, we have

$$
e_{k l m} P_{k i} P_{l j} P_{m n}=-e_{k l m} P_{k 1} P_{l 2} P_{m 3}=-\operatorname{det}(P)=\operatorname{det}(P) e_{i j n} .
$$

In all cases we get $e_{k l m} P_{k i} P_{l j} P_{m n}=\operatorname{det}(P) e_{i j n}$, and hence by $(3.16)$

$$
\left[P^{T} \mathcal{E}(P \eta) P\right]_{i j}=\operatorname{det}(P) e_{i j n} \eta_{n}=\operatorname{det}(P)[\mathcal{E}(\eta)]_{i j} \quad \text { for any } i, j \in\{1,2,3\},
$$

i.e. $P^{T} \mathcal{E}(P \eta) P=\operatorname{det}(P) \mathcal{E}(\eta)$. This combined with the equality $\operatorname{Cof}(P)^{T}=\operatorname{det}(P) P^{-1}$ thus yields

$$
P^{T} \mathcal{E}(\xi) P=\mathcal{E}\left(\operatorname{det}(P) P^{-1} \xi\right)=\mathcal{E}\left(\operatorname{Cof}(P)^{T} \xi\right),
$$

which proves the claim for any invertible matrix $P$. The result holds for any matrix $P$ by a density argument.

Proof of Theorem 3.6. Let $P^{\varepsilon}$ be a corrector associated with the resistivity $\sigma^{\varepsilon}$. By Lemma 3.8 we have for any $h \in O$,

$$
\left(P^{\varepsilon}\right)^{T} \mathcal{E}\left(S^{\varepsilon} h\right) P^{\varepsilon}=\mathcal{E}\left(\operatorname{Cof}\left(P^{\varepsilon}\right)^{T} S^{\varepsilon} h\right) .
$$

Therefore, using the fact that the first-order terms of (3.7) and (3.11) are respectively $\mathcal{E}\left(S^{\varepsilon} h\right)$ and $\mathcal{E}\left(S^{*} h\right)$, we deduce from $(2.8)$ the convergences

$$
\forall h \in O, \quad \mathcal{E}\left(\operatorname{Cof}\left(P^{\varepsilon}\right)^{T} S^{\varepsilon} h\right) \longrightarrow \mathcal{E}\left(S^{*} h\right) \quad \text { in } \mathcal{D}^{\prime}(\Omega)^{3 \times 3} .
$$

Since $\mathcal{E}$ is an invertible linear mapping, the desired convergence (3.13) thus follows from the former ones. Now, the convergence (3.14) is a straightforward consequence of the convergence (3.13) combined with relations (3.12) and the multiplicativity of the cofactor matrix.

Proof of Proposition 3.5. By the first-order expansions (3.7) and (3.9) we have for any $h \in O$ and any $t>0$ small enough,

$$
\begin{aligned}
0 & =-I+\sigma^{\varepsilon}(t h) \rho^{\varepsilon}(t h)=-I+\left(\sigma^{\varepsilon}+\mathcal{E}\left(t S^{\varepsilon} h\right)\right)\left(\rho^{\varepsilon}+\mathcal{E}\left(t R^{\varepsilon} h\right)\right)+o_{L^{\infty}(\Omega)^{3 \times 3}}(t h) \\
& =t \sigma^{\varepsilon} \mathcal{E}\left(R^{\varepsilon} h\right)+t \mathcal{E}\left(S^{\varepsilon} h\right)\left(\sigma^{\varepsilon}\right)^{-1}+o_{L^{\infty}(\Omega)^{3 \times 3}}(t h) .
\end{aligned}
$$

Then, dividing by $t$ the previous equality and letting $t$ tend to zero we obtain by the symmetry of $\sigma^{\varepsilon}$

$$
\mathcal{E}\left(S^{\varepsilon} h\right)=-\sigma^{\varepsilon} \mathcal{E}\left(R^{\varepsilon} h\right) \sigma^{\varepsilon}=-\left(\sigma^{\varepsilon}\right)^{T} \mathcal{E}\left(R^{\varepsilon} h\right) \sigma^{\varepsilon} .
$$

Therefore, by Lemma 3.8 we get

$$
\mathcal{E}\left(S^{\varepsilon} h\right)=-\varepsilon\left(\operatorname{Cof}\left(\sigma^{\varepsilon}\right)^{T} R^{\varepsilon} h\right)=-\varepsilon\left(\operatorname{Cof}\left(\sigma^{\varepsilon}\right) R^{\varepsilon} h\right),
$$

which yields the first equality of (3.12) using the invertibility of $\mathcal{E}$.

The proof of the second one is quite similar starting from (3.10) and (3.11). 


\section{Properties of the homogenized Hall matrix}

\subsection{Statement of the properties}

Let $\Omega$ be a bounded open subset of $\mathbb{R}^{3}$, let $O$ be a ball of $\mathbb{R}^{3}$ centered at 0 , and let $\alpha, \beta>0$. We consider a conductivity sequence $\sigma^{\varepsilon}(h)$, for $\varepsilon>0$ and $h \in O$, in $\mathcal{M}(\alpha, \beta ; \Omega)$ which is associated with the resistivity sequence $\rho^{\varepsilon}(h):=\left[\sigma^{\varepsilon}(h)\right]^{-1}$ in $\mathcal{M}\left(\beta^{-1}, \alpha^{-1} ; \Omega\right)$. We make the assumptions of Section 3.1 to ensure that the Hall matrix $R^{\varepsilon}$ and its homogenized $R^{*}$ defined by (3.7) and (3.11) satisfy convergence (3.14).

We have the following result of stability satisfied by the Hall matrices $R^{\varepsilon}, R^{\varepsilon}$ and the $S$-matrices $S^{\varepsilon}, S^{\varepsilon}:$

Proposition 4.1. Assume that there exist two matrix-valued functions $\sigma, R$ in $L^{\infty}(\Omega)^{3 \times 3}$ such that $\sigma^{\varepsilon}=\sigma$ and $R^{\varepsilon}=R$ a.e. in $\Omega$. Then, we have $R^{*}=R$ a.e. in $\Omega$.

Assume that there exists a matrix-valued function $S$ in $L^{\infty}(\Omega)^{3 \times 3}$ such that $S^{\varepsilon}=S$ a.e. in $\Omega$. Then, we have $S^{*}=S$ a.e. in $\Omega$.

Note that the previous result about the homogenized $S$-matrix $S^{*}$ was already known to Stroud and Bergman [15].

Proof. The first part of Proposition 4.1 is immediate since we then have $\sigma^{\varepsilon}=\sigma^{*}=\sigma$ and $P^{\varepsilon}=I$ in the convergence (3.14). Let us now prove the second part. Let $P^{\varepsilon}:=D U^{\varepsilon}$ be the corrector associated with $\sigma^{\varepsilon}$, where $U^{\varepsilon}$ is the solution of equation (2.3) with $A^{\varepsilon}:=\sigma^{\varepsilon}$. The continuity of the null Lagrangians (see e.g. Corollary 2.8 p. 179 of [7]) combined with the convergence of $P^{\varepsilon}$ to $I$ in $L^{2}(\Omega)^{3 \times 3}$, implies that the cofactor matrix $\operatorname{Cof}\left(P^{\varepsilon}\right)$ converges to $I$ in $\mathcal{D}^{\prime}(\Omega)^{3 \times 3}$. Moreover, thanks to the Meyers estimate [10] the sequence $P^{\varepsilon}$ is also bounded in $L_{\text {loc }}^{p}(\Omega)^{3 \times 3}$, for some $p>2$. Therefore, the sequence $\operatorname{Cof}\left(P^{\varepsilon}\right)$ is bounded in $L_{\text {loc }}^{p / 2}(\Omega)^{3 \times 3}$ and weakly converges to $I$ in $L_{\text {loc }}^{p / 2}(\Omega)^{3 \times 3}$. This combined with the convergence (3.13) and the fact that $S \in L^{\infty}(\Omega)^{3 \times 3}$ yields

$$
\operatorname{Cof}\left(P^{\varepsilon}\right)^{T} S^{\varepsilon}=\operatorname{Cof}\left(P^{\varepsilon}\right)^{T} S \longrightarrow S=S^{*} \quad \text { weakly in } L_{\text {loc }}^{p / 2}(\Omega)^{3 \times 3},
$$

which concludes the proof.

In addition to this stability property, the two-dimensional effective Hall coefficient keeps the sign of the Hall coefficient associated with the microstructure (see [4]). However, this positivity property is not preserved in dimension three as shown by the following result:

Theorem 4.2. There exists a microstructure with conductivity $\sigma^{\varepsilon}(h):=\sigma^{\varepsilon}+\mathcal{E}\left(S^{\varepsilon} h\right)$, such that the conductivity $\sigma^{\varepsilon}$ is isotropic and the Hall matrix $R^{\varepsilon}$ is positive isotropic a.e. in $\Omega$, while the effective Hall matrix $R^{*}$ is a constant negative isotropic matrix.

Remark 4.3. The fact that the Hall coefficient keeps its sign in the two-dimensional homogenization process is strongly linked to the nonnegativity of the corrector's determinant $\operatorname{det}\left(D U^{\varepsilon}\right)$ defined by $(2.3)$. This nonnegativity is proved in [2] assuming that the homogenized matrix is constant, which holds in particular for periodic microstructures. By contrast, we proved in [5] that the corrector's determinant may change sign in dimension three (see also [1] using a similar geometry for a different purpose). Using a suitable modification of the geometry of the counter-example from [5], we will build a microstructure which establishes the claim of Theorem 4.2.

\subsection{Proof of Theorem 4.2}

The proof of Theorem 4.2 is divided into three steps. In the first step we build a microstructure with cubic symmetry in which the electric field in some areas points in the opposite direction to the applied field. In the second step we define an isotropic $S$-matrix $S^{\varepsilon}=s_{\varepsilon} I$ in such a way that the homogenized $S$-matrix $S^{*}=s_{*} I$ is also isotropic. In the third and final step we prove that the parameters of the 


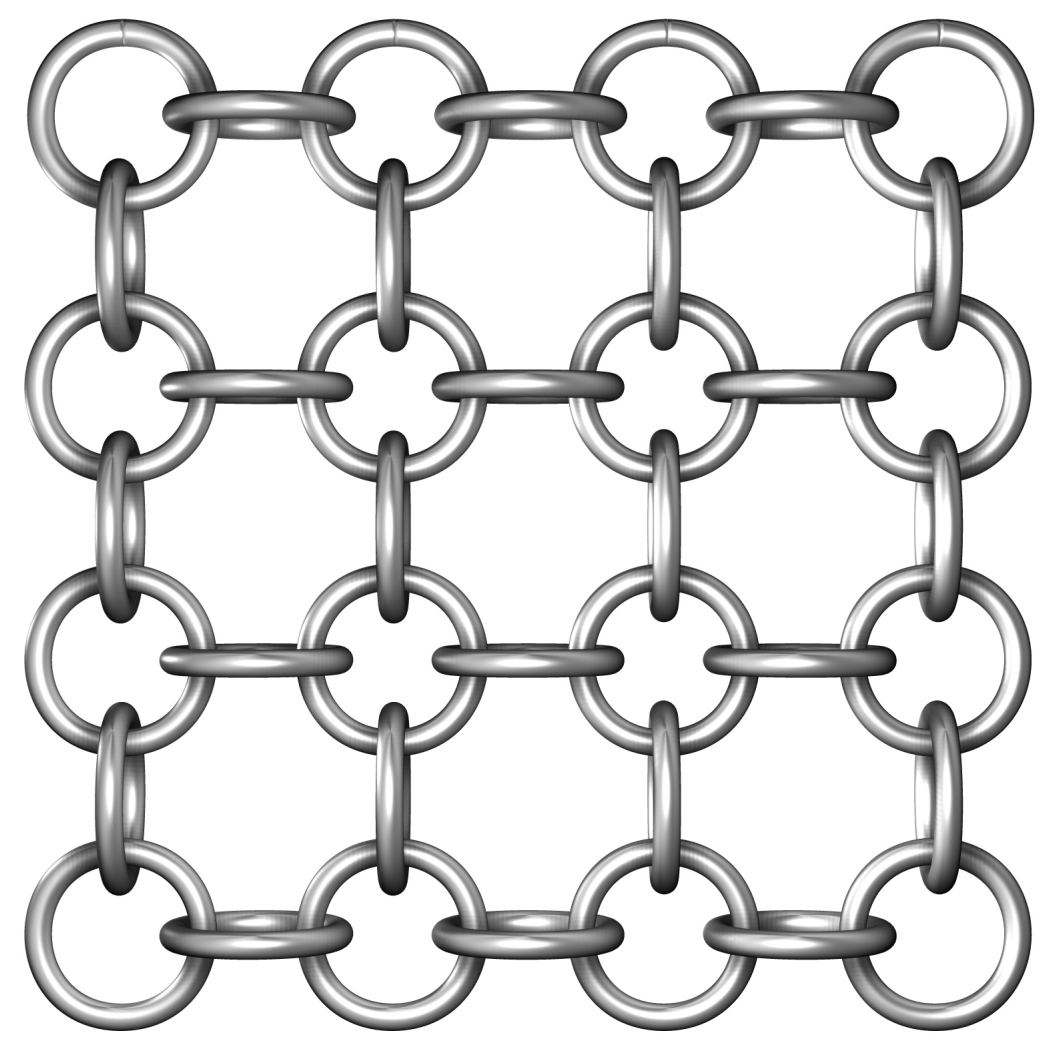

Figure 1: A sheet of chain mail. Reprinted with kind permission from Dylon Whyte, http://artofchainmail.com/patterns/japanese/hitoye_gusari.html, copyright 1998-2004.

model can be chosen so that the $s$-coefficients $s_{\varepsilon}$ and $s_{*}$ have opposite signs, which completes the proof. Indeed, due to the cubic symmetry and the local isotropy of $\sigma^{\varepsilon}$ the homogenized conductivity $\sigma^{*}$ is known to be isotropic: this is obvious since the eigenspaces of $\sigma^{*}$ must inherit the cubic symmetry and cannot have preferred directions. Therefore, by virtue of relations (3.12) the isotropy and the change of sign satisfied by the $S$-matrices $S^{\varepsilon}, S^{*}$ also hold for the Hall matrices $R^{\varepsilon}, R^{*}$.

First step: The microstructure and the induced symmetry properties.

The microstructure is defined by the three following steps:

- First, we start from the geometry of [5] restricted to the strip $(-1,1) \times \mathbb{R}^{2}$. It consists of separate chains parallel to the $x_{3}$ axis, with the chain axes spaced a distance 2 apart. Each link is isometric to a closed torus of outer radius $R \in\left(\frac{1}{2}, 1\right)$ and of inner radius $r \in\left(\frac{1}{2}, R\right)$. The links each have a central axis alternatively parallel to the $x_{1}$ and $x_{2}$ axes. Those links oriented with their central axes parallel to the $x_{1}$ axis have centers at the points $(0, \ell, m)$, where $\ell$ and $m$ are even integers, while those links oriented with their central axes parallel to the $x_{2}$ axis have centers at the points $(0, \ell, m+1)$.

- Second, in the strip $(-1,1) \times \mathbb{R}^{2}$ we add to the previous array of chains a set of orthogonal cross links, each with the same dimensions as the original links and having a central axis parallel to $x_{3}$, and centered at the points $(0, \ell+1, m)$. In this way we form a sheet $\Sigma$ of chain mail (as in Middle Age armor) with square symmetry, as shown in figure 1. The sheet $\Sigma$ is 2-periodic with respect to $x_{2}, x_{3}$, symmetric with respect to the planes $x_{1} O x_{2}, x_{1} O x_{3}$, and invariant by rotation of $90^{\circ}$ in the plane $x_{2} O x_{3}$.

- Third, we construct cubic chain mail defined by the following union of appropriately translated 


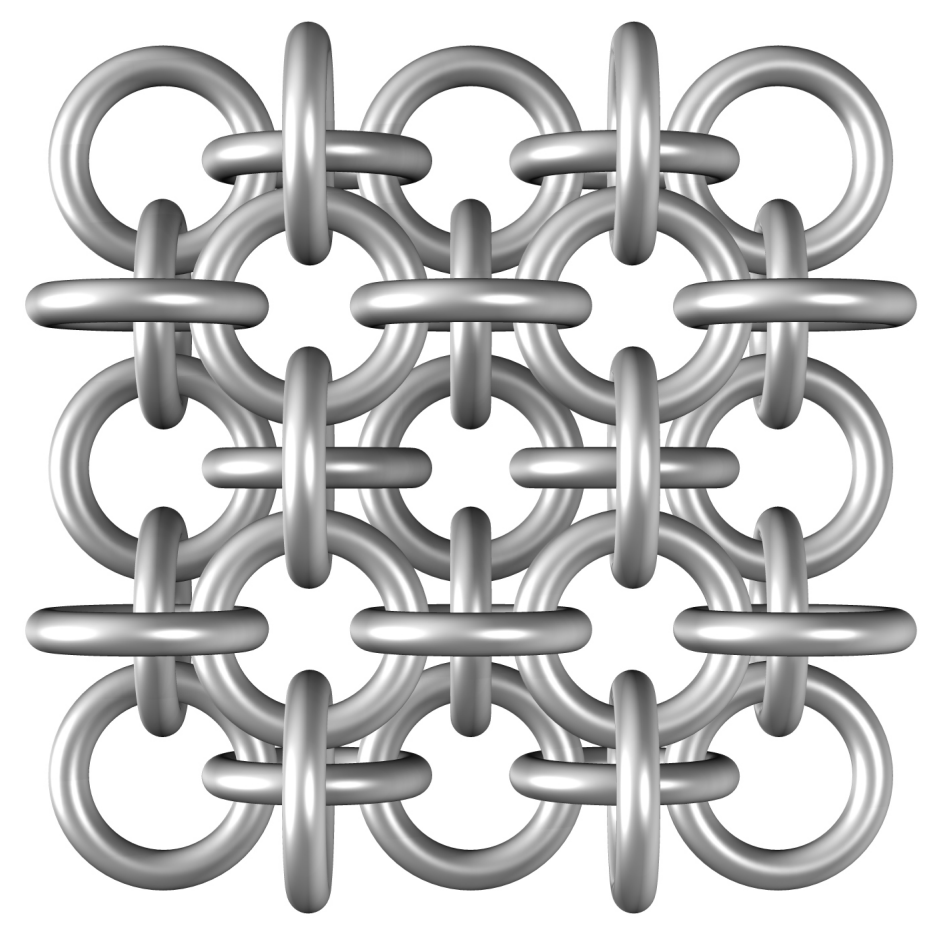

Figure 2: Two sheets of cubic chain mail. Reprinted with kind permission from Dylon Whyte, http://artofchainmail.com/patterns/japanese/hitoye_gusari.html, copyright 1998-2004.

sheets $\Sigma$ :

$$
Q_{\#}:=\bigcup_{k \in \mathbb{Z}}[(k, k, k)+\Sigma] .
$$

To convey the idea, figure 2 represents two sheets of $Q_{\#}$ made of a subset of $\Sigma \cup[(1,1,1)+\Sigma]$. Set $Y:=(-1,1)^{3}$. The set $Q_{\#}$ is closed, $Y$-periodic and has cubic symmetry. Within the cell $Y$ figure 3 represents the structure $Q_{\#} \cap Y$ of $Q_{\#}$ (for the sake of clarity only the boundary of the rings is represented and some connected components of $Q_{\#} \cap Y$ are not), and figure 4 represents two cross-sections of $Q_{\#} \cap Y$.

In the absence of a magnetic field the conductivity associated with this microstructure is defined, for a fixed $\kappa>1$, by

$$
\sigma^{\kappa, \varepsilon}(x):=\sigma^{\kappa}\left(\frac{x}{\varepsilon}\right) \quad \text { where } \quad \sigma^{\kappa}(y):=\left\{\begin{aligned}
\kappa I & \text { if } y \in Q_{\#} \\
I & \text { elsewhere. }
\end{aligned}\right.
$$

According to Example 2.6 the corrector associated with $\sigma^{\kappa, \varepsilon}$ is defined by

$$
P^{\varepsilon}(x):=D U^{\kappa}\left(\frac{x}{\varepsilon}\right), \quad \text { for } x \in \Omega,
$$

where $U^{\kappa}$ is the zero $Y$-average solution of equation (2.4) with $A:=\sigma^{\kappa}$. Following the arguments of [5] we can prove that $U^{\kappa}$ strongly converges in $H_{\text {loc }}^{1}\left(\mathbb{R}^{3}\right)$, as $\kappa \rightarrow+\infty$, to the zero $Y$-average function $U$ which is the solution of the equation (see figure 4)

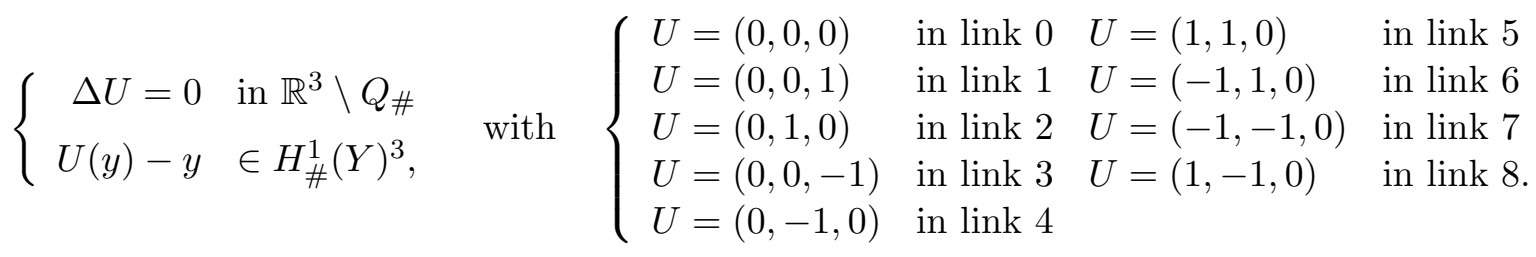




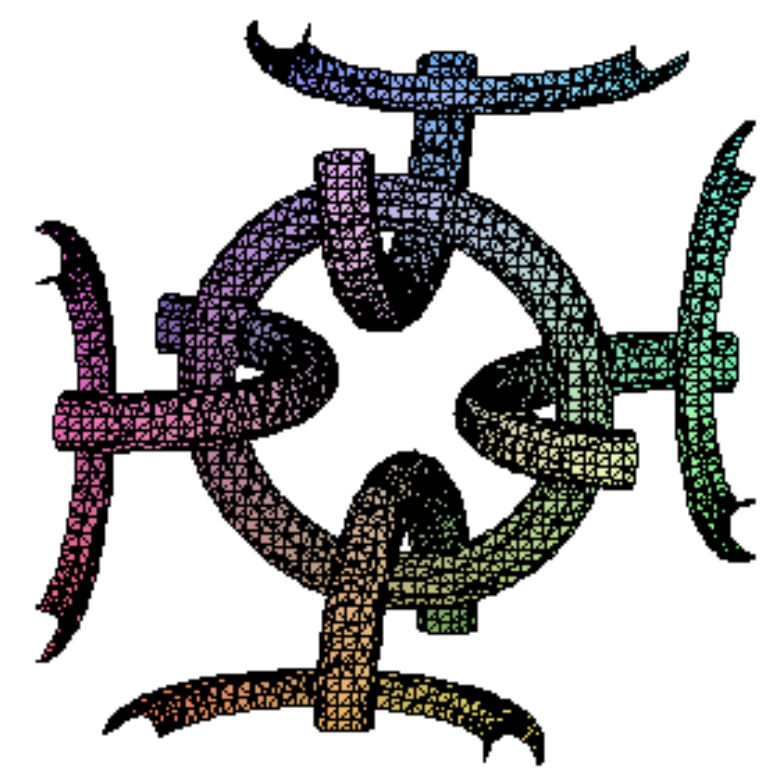

Figure 3: The cubic chain mail within the cell $(-1,1)^{3}$, missing some connected components

Now, let us show the symmetry properties satisfied by the function $U^{\kappa}$ as a consequence of the symmetries satisfied by the set $Q_{\#}$ and the function $\sigma^{\kappa}$. On the one hand, we can check that $Q_{\#}$ and $\sigma^{\kappa}$ are left invariant by the orthogonal transformations

$$
\theta_{1}(x):=\left(x_{1}, x_{3}, x_{2}\right) \text { and } \theta_{2}(x):=\left(x_{3}, x_{2}+1, x_{1}\right),
$$

and thus by the compound transformations $\theta:=\theta_{2} \circ \theta_{1}$ and $\theta \circ \theta$ given by

$$
\theta(x)=\left(x_{2}, x_{3}+1, x_{1}\right) \text { and }(\theta \circ \theta)(x)=\left(x_{3}+1, x_{1}+1, x_{2}\right) .
$$

This combined with the definition (2.4) of $U^{\kappa}$ and the isotropy of $\sigma^{\kappa}$ leads us to the following relations, for a.e. $x \in \mathbb{R}^{3}$,

$$
\left\{\begin{array}{l}
u_{1}^{\kappa}(x)=u_{3}^{\kappa}\left(x_{2}, x_{3}+1, x_{1}\right)=u_{2}^{\kappa}\left(x_{3}+1, x_{1}+1, x_{2}\right) \\
u_{2}^{\kappa}(x)=u_{1}^{\kappa}\left(x_{2}, x_{3}+1, x_{1}\right)=u_{3}^{\kappa}\left(x_{3}+1, x_{1}+1, x_{2}\right) \\
u_{3}^{\kappa}(x)=u_{2}^{\kappa}\left(x_{2}, x_{3}+1, x_{1}\right)=u_{1}^{\kappa}\left(x_{3}+1, x_{1}+1, x_{2}\right) .
\end{array}\right.
$$

These properties easily imply that the cofactor matrix $\operatorname{Cof}\left(D U^{\kappa}\right)=\left[c_{i j}^{\kappa}\right]_{1 \leq i, j \leq 3}$ satisfies the following transformations, for a.e. $x \in \mathbb{R}^{3}$,

$$
\begin{aligned}
\operatorname{Cof}\left(D U^{\kappa}\right)\left(x_{2}, x_{3}+1, x_{1}\right) & =\left(\begin{array}{ccc}
c_{22}^{\kappa} & c_{23}^{\kappa} & c_{21}^{\kappa} \\
c_{32}^{\kappa} & c_{33}^{\kappa} & c_{31}^{\kappa} \\
c_{12}^{\kappa} & c_{13}^{\kappa} & c_{11}^{\kappa}
\end{array}\right)(x) \\
\operatorname{Cof}\left(D U^{\kappa}\right)\left(x_{3}+1, x_{1}+1, x_{2}\right) & =\left(\begin{array}{ccc}
c_{33}^{\kappa} & c_{31}^{\kappa} & c_{32}^{\kappa} \\
c_{13}^{\kappa} & c_{11}^{\kappa} & c_{12}^{\kappa} \\
c_{23}^{\kappa} & c_{21}^{\kappa} & c_{22}^{\kappa}
\end{array}\right)(x),
\end{aligned}
$$

where the elements on the right hand sides are obtained from the elements of $\operatorname{Cof}\left(D U^{\kappa}\right)(x)$ according to the permutations $(1,2,3) \rightarrow(2,3,1),(1,2,3) \rightarrow(3,1,2)$ respectively. 

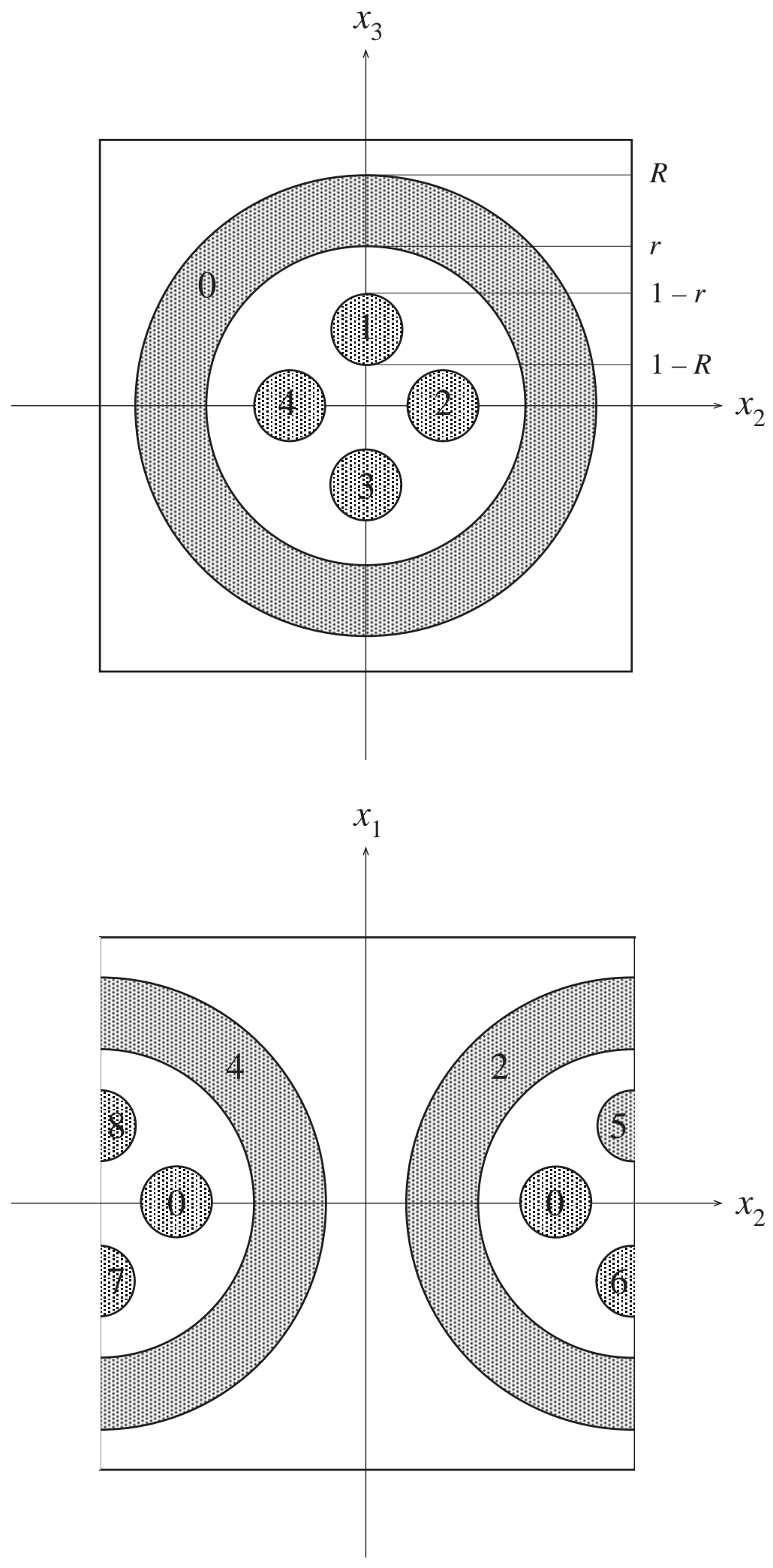

Figure 4: Sections $x_{2} O x_{3}$ and $x_{1} O x_{2}$ of the period cell: 0 is an entire link, 1,2,3,4 are half-links, and $5,6,7,8$ are quarter-links. 
On the other hand, due to the symmetries of $Q_{\#}$ and $\sigma^{\kappa}$ (4.3) one can check that the $i$-th coordinate $u_{i}^{\kappa}$ of $U^{K}$ is odd with respect to the variable $x_{i}$ and even with respect to $x_{j}$ for $j \neq i$. This implies that, for a.e. $x \in \mathbb{R}^{3}$,

$$
D U^{\kappa}\left(-x_{1}, x_{2}, x_{3}\right)=\left(\begin{array}{rrr}
\frac{\partial u_{1}^{\kappa}}{\partial x_{1}} & -\frac{\partial u_{2}^{\kappa}}{\partial x_{1}} & -\frac{\partial u_{3}^{\kappa}}{\partial x_{1}} \\
-\frac{\partial u_{1}^{\kappa}}{\partial x_{2}} & \frac{\partial u_{2}^{\kappa}}{\partial x_{2}} & \frac{\partial u_{3}^{\kappa}}{\partial x_{2}} \\
-\frac{\partial u_{1}^{\kappa}}{\partial x_{3}} & \frac{\partial u_{2}^{\kappa}}{\partial x_{3}} & \frac{\partial u_{3}^{\kappa}}{\partial x_{3}}
\end{array}\right)(x), \quad D U^{\kappa}\left(x_{1},-x_{2}, x_{3}\right)=\left(\begin{array}{rrr}
\frac{\partial u_{1}^{\kappa}}{\partial x_{1}} & -\frac{\partial u_{2}^{\kappa}}{\partial x_{1}} & \frac{\partial u_{3}^{\kappa}}{\partial x_{1}} \\
-\frac{\partial u_{1}^{\kappa}}{\partial x_{2}} & \frac{\partial u_{2}^{\kappa}}{\partial x_{2}} & -\frac{\partial u_{3}^{\kappa}}{\partial x_{2}} \\
\frac{\partial u_{1}^{\kappa}}{\partial x_{3}} & -\frac{\partial u_{2}^{\kappa}}{\partial x_{3}} & \frac{\partial u_{3}^{\kappa}}{\partial x_{3}}
\end{array}\right)(x),
$$

and hence the cofactor matrix $\operatorname{Cof}\left(D U^{\kappa}\right)$ satisfies the same changes of sign

$$
\begin{aligned}
\operatorname{Cof}\left(D U^{\kappa}\right)\left(-x_{1}, x_{2}, x_{3}\right) & =\left(\begin{array}{rrr}
c_{11}^{\kappa} & -c_{12}^{\kappa} & -c_{13}^{\kappa} \\
-c_{21}^{\kappa} & c_{22}^{\kappa} & c_{23}^{\kappa} \\
-c_{31}^{\kappa} & c_{32}^{\kappa} & c_{33}^{\kappa}
\end{array}\right)(x), \\
\operatorname{Cof}\left(D U^{\kappa}\right)\left(x_{1},-x_{2}, x_{3}\right) & =\left(\begin{array}{rrr}
c_{11}^{\kappa} & -c_{12}^{\kappa} & c_{13}^{\kappa} \\
-c_{21}^{\kappa} & c_{22}^{\kappa} & -c_{23}^{\kappa} \\
c_{31}^{\kappa} & -c_{32}^{\kappa} & c_{33}^{\kappa}
\end{array}\right)(x) .
\end{aligned}
$$

Second step: Construction of the isotropic Hall matrices.

Let $\tau \in(1-r, r)$ be a parameter which will be chosen more precisely in the third step. Consider the three points in $\mathbb{R}^{3} \backslash Q_{\#}$ defined by

$$
\hat{x}:=(0,0, \tau), \quad \hat{y}:=(0, \tau+1,0) \quad \text { and } \quad \hat{z}:=(\tau+1,1,0),
$$

and, for $\delta \in\left(\frac{1}{2}, 1\right)$, the matrix defined by

$$
S_{\delta}^{\kappa}:=f_{B(\hat{x}, \delta)} \operatorname{Cof}\left(D U^{\kappa}\right)^{T}(x) d x+f_{B(\hat{y}, \delta)} \operatorname{Cof}\left(D U^{\kappa}\right)^{T}(y) d y+f_{B(\hat{z}, \delta)} \operatorname{Cof}\left(D U^{\kappa}\right)^{T}(z) d z,
$$

where $B(\hat{x}, \delta)$ denotes the open ball of center $\hat{x}$ and radius $\delta$.

Let us prove that the $S$-matrix $S_{\delta}^{\kappa}$ is isotropic. On the one hand, the symmetry of the ball $B(\hat{x}, \delta)$ with respect to the plane $x_{2} O x_{3}$ combined with relations (4.11) yields

$$
\int_{B(\hat{x}, \delta)} \operatorname{Cof}\left(D U^{\kappa}\right)(x) d x=2 \int_{B(\hat{x}, \delta) \cap\left\{x_{1}>0\right\}}\left(\begin{array}{ccc}
c_{11}^{\kappa} & 0 & 0 \\
0 & c_{22}^{\kappa} & c_{23}^{\kappa} \\
0 & c_{32}^{\kappa} & c_{33}^{\kappa}
\end{array}\right)(x) d x .
$$

Then, using the symmetry of the half-ball $B(\hat{x}, \delta) \cap\left\{x_{1}>0\right\}$ with respect to the plane $x_{1} O x_{3}$, we get

$$
\int_{B(\hat{x}, \delta)} \operatorname{Cof}\left(D U^{\kappa}\right)(x) d x=4 \int_{B(\hat{x}, \delta) \cap\left\{x_{1}, x_{2}>0\right\}}\left(\begin{array}{ccc}
c_{11}^{\kappa} & 0 & 0 \\
0 & c_{22}^{\kappa} & 0 \\
0 & 0 & c_{33}^{\kappa}
\end{array}\right)(x) d x .
$$

On the other hand, making the changes of variables $y=\left(x_{2}, x_{3}+1, x_{1}\right), z=\left(x_{3}+1, x_{1}+1, x_{2}\right)$ in the balls $B(\hat{y}, \delta), B(\hat{z}, \delta)$ respectively, and using the relations (4.9) we have

$$
\begin{aligned}
\int_{B(\hat{y}, \delta)} \operatorname{Cof}\left(D U^{\kappa}\right)(y) d y & =\int_{B(\hat{x}, \delta)}\left(\begin{array}{ccc}
c_{22}^{\kappa} & c_{23}^{\kappa} & c_{21}^{\kappa} \\
c_{32}^{\kappa} & c_{33}^{\kappa} & c_{31}^{\kappa} \\
c_{12}^{\kappa} & c_{13}^{\kappa} & c_{11}^{\kappa}
\end{array}\right)(x) d x, \\
\int_{B(\hat{z}, \delta)} \operatorname{Cof}\left(D U^{\kappa}\right)(z) d z & =\int_{B(\hat{x}, \delta)}\left(\begin{array}{ccc}
c_{33}^{\kappa} & c_{31}^{\kappa} & c_{32}^{\kappa} \\
c_{13}^{\kappa} & c_{11}^{\kappa} & c_{12}^{\kappa} \\
c_{23}^{\kappa} & c_{21}^{\kappa} & c_{22}^{\kappa}
\end{array}\right)(x) d x .
\end{aligned}
$$

This combined with relations (4.9) yields, similarly to (4.15),

$$
\begin{aligned}
& \int_{B(\hat{y}, \delta)} \operatorname{Cof}\left(D U^{\kappa}\right)(y) d y=4 \int_{B(\hat{x}, \delta) \cap\left\{x_{1}, x_{2}>0\right\}}\left(\begin{array}{ccc}
c_{22}^{\kappa} & 0 & 0 \\
0 & c_{33}^{\kappa} & 0 \\
0 & 0 & c_{11}^{\kappa}
\end{array}\right)(x) d x \\
& \int_{B(\hat{z}, \delta)} \operatorname{Cof}\left(D U^{\kappa}\right)(z) d z=4 \int_{B(\hat{x}, \delta) \cap\left\{x_{1}, x_{2}>0\right\}}\left(\begin{array}{ccc}
c_{33}^{\kappa} & 0 & 0 \\
0 & c_{11}^{\kappa} & 0 \\
0 & 0 & c_{22}^{\kappa}
\end{array}\right)(x) d x .
\end{aligned}
$$


Therefore, from the definition (4.13) of $S_{\delta}^{\kappa},(4.15)$ and (4.17) we deduce that

$$
S_{\delta}^{\kappa}=s_{\delta}^{\kappa} I, \quad \text { where } \quad s_{\delta}^{\kappa}:=f_{B(\hat{x}, \delta) \cap\left\{x_{1}, x_{2}>0\right\}} \operatorname{tr}\left(\operatorname{Cof}\left(D U^{\kappa}\right)\right)(x) d x
$$

Now, consider the microstructure with conductivity $\sigma^{\kappa, \varepsilon}(h)=\sigma^{\kappa, \varepsilon}+\mathcal{E}\left(S^{\varepsilon} h\right)$, where $\sigma^{\kappa, \varepsilon}$ is defined by (4.3) and the $S$-matrix $S^{\varepsilon}$ is defined, for a fixed $\gamma \in(0,1)$ and a.e. $x \in \Omega$, by

$$
S^{\varepsilon}(x):=s_{\varepsilon}(x) I, \quad s_{\varepsilon}(x):=s_{\#}\left(\frac{x}{\varepsilon}\right), \quad s_{\#}:=\gamma+(1-\gamma)\left(1_{B(\hat{x}, \delta)}^{\#}+1_{B(\hat{y}, \delta)}^{\#}+1_{B(\hat{z}, \delta)}^{\#}\right),
$$

where $1_{E}^{\#}$ denotes the characteristic function of the set $E$, extended by $Y$-periodicity to the whole space $\mathbb{R}^{3}$. In other words, the $s$-coefficient $s_{\varepsilon}$ is equal to 1 in the set composed by the three balls $B(\varepsilon \hat{x}, \varepsilon \delta), B(\varepsilon \hat{y}, \varepsilon \delta), B(\varepsilon \hat{z}, \varepsilon \delta)$ repeated by $\varepsilon Y$-periodicity in $\mathbb{R}^{3}$, and to $\gamma$ elsewhere. Due to the convergence (3.14) combined with the $\varepsilon Y$-periodicity of the corrector $P^{\varepsilon}$ (4.4) and of the $S$-matrix $S^{\varepsilon}$ (4.19), the homogenized $S$-matrix $S^{*}$ is given by the weak convergence

$$
\operatorname{Cof}\left(P^{\varepsilon}\right)^{T} S^{\varepsilon} \longrightarrow S^{*}=f_{Y} s_{\#}(x) \operatorname{Cof}\left(D U^{\kappa}\right)^{T}(x) d x \quad \text { weakly in } L^{1}(\Omega)^{3 \times 3} .
$$

Since Cof is a null Lagrangian and $D U^{\kappa}$ is $Y$-periodic, we have

$$
f_{Y} \operatorname{Cof}\left(D U^{\kappa}\right)(x) d x=I
$$

Therefore, by the definition (4.13) of $S_{\delta}^{\kappa}$ we get

$$
S^{*}=\gamma I+(1-\gamma) \frac{|B(\hat{x}, \delta)|}{|Y|} S_{\delta}^{\kappa}=\gamma I+(1-\gamma) \frac{\pi}{6} \delta^{3} S_{\delta}^{\kappa} .
$$

By the definition $(4.18)$ of $s_{\delta}^{\kappa}$ the $S$-matrix $S^{*}$ also reads as

$$
S^{*}=s_{*} I, \quad \text { where } \quad s_{*}:=\frac{\pi}{6} \delta^{3} s_{\delta}^{\kappa}+\gamma\left(1-\frac{\pi}{6} \delta^{3} s_{\delta}^{\kappa}\right) .
$$

Let us conclude. The definition (4.19) clearly shows that $S^{\varepsilon}$ is positive isotropic a.e. in $\Omega$. Therefore, by virtue of (4.23) it remains to prove that $r, R, \kappa, \delta$ can be chosen in such a way that $s_{\delta}^{\kappa}<0$. This is the aim of the third step.

Remark 4.4. The previous analysis is sufficient to ensure the isotropy of the homogenized $S$ matrix $S^{*}$. In some sense the choice of the three points (4.12) is the minimal way to obtain isotropy, since these points allow us to get the permutations of the diagonal coefficients of $\operatorname{Cof}\left(D U^{\kappa}\right)$ in $(4.17)$. However, the microstructure (4.19) of the $S$-matrix $S^{\varepsilon}$ has not the cubic symmetry of the conductivity matrix (4.3). In order to obtain this symmetry we can consider the three extra points

$$
\hat{x}^{\prime}:=(0,0,-\tau), \quad \hat{y}^{\prime}:=(0,-\tau+1,0), \quad \hat{z}^{\prime}:=(-\tau+1,1,0),
$$

and the isotropic $S$-matrix $S_{\varepsilon}^{\prime}:=s_{\#}^{\prime}\left(\frac{x}{\varepsilon}\right) I$ now defined with six balls by (compare to (4.19))

$$
s_{\#}^{\prime}:=\gamma+(1-\gamma)\left(1_{B(\hat{x}, \delta)}^{\#}+1_{B(\hat{y}, \delta)}^{\#}+1_{B(\hat{z}, \delta)}^{\#}+1_{B\left(\hat{x}^{\prime}, \delta\right)}^{\#}+1_{B\left(\hat{y}^{\prime}, \delta\right)}^{\#}+1_{B\left(\hat{z}^{\prime}, \delta\right)}^{\#}\right) .
$$

Then, the matrix-valued function $\operatorname{Cof}\left(P^{\varepsilon}\right)^{T} S_{\varepsilon}^{\prime}$ has cubic symmetry. Therefore, according to the principle that any effective tensor inherits the symmetries of the associated microstructure (this can be rigorously checked for example by arguments similar to the above analysis) the constant homogenized $S$-matrix $S_{*}^{\prime}$ as weak limit of $\operatorname{Cof}\left(P^{\varepsilon}\right)^{T} S_{\varepsilon}^{\prime}$ (see (4.20)) is necessarily isotropic, i.e. $S_{*}^{\prime}:=s_{*}^{\prime} I$. By linearity the trace of $\operatorname{Cof}\left(P^{\varepsilon}\right)^{T} S_{\varepsilon}^{\prime}$ weakly converges to the trace of $S_{*}^{\prime}$, which leads to

$$
3 s_{*}^{\prime}=3 \gamma+\frac{(1-\gamma)}{|Y|} \int_{B(\hat{x}, \delta) \cup B(\hat{y}, \delta) \cup B(\hat{z}, \delta) \cup B\left(\hat{x}^{\prime}, \delta\right) \cup B\left(\hat{y}^{\prime}, \delta\right) \cup B\left(\hat{z}^{\prime}, \delta\right)} \operatorname{tr}\left(\operatorname{Cof}\left(D U^{\kappa}\right)\right)(x) d x .
$$


Again using the cubic symmetry and the definition (4.18) of $s_{\delta}^{\kappa}$, we obtain

$$
s_{*}^{\prime}=\gamma+\frac{\pi}{3} \delta^{3}(1-\gamma) f_{B(\hat{x}, \delta)} \operatorname{tr}\left(\operatorname{Cof}\left(D U^{\kappa}\right)\right)(x) d x=\frac{\pi}{3} \delta^{3} s_{\delta}^{\kappa}+\gamma\left(1-\frac{\pi}{3} \delta^{3} s_{\delta}^{\kappa}\right) .
$$

Third step: Negativity of the homogenized $s$-coefficient $s_{*}$.

Since the sequence $D U^{\kappa}$ strongly converges to $D U(4.5)$ in $L_{\text {loc }}^{2}\left(\mathbb{R}^{3}\right)$, and since the function $D U$ is continuous at the point $\hat{x}$ (by the harmonicity of $U$ in $\mathbb{R}^{3} \backslash Q_{\#}$ ), we have by definition (4.18)

$$
\lim _{\delta \rightarrow 0}\left(\lim _{\kappa \rightarrow+\infty} s_{\delta}^{\kappa}\right)=\operatorname{tr}(\operatorname{Cof}(D U))(\hat{x})
$$

Moreover, since the function $u_{i}$ is even with respect to the variable $x_{j}$ for $j \neq i$, we have $\frac{\partial u_{i}}{\partial x_{j}}(\hat{x})=0$ for any $j=1,2$ and $i \neq j$, hence

$$
\lim _{\delta \rightarrow 0}\left(\lim _{\kappa \rightarrow+\infty} s_{\delta}^{\kappa}\right)=\frac{\partial u_{1}}{\partial x_{1}}(\hat{x}) \frac{\partial u_{2}}{\partial x_{2}}(\hat{x})+\frac{\partial u_{1}}{\partial x_{1}}(\hat{x}) \frac{\partial u_{3}}{\partial x_{3}}(\hat{x})+\frac{\partial u_{2}}{\partial x_{2}}(\hat{x}) \frac{\partial u_{3}}{\partial x_{3}}(\hat{x}) .
$$

Let us prove that there exists $r \in\left(\frac{1}{2}, 1\right)$ such that this limit is negative.

On the one hand, since $r>1-r, u_{3}(0,0, r)=0$ and $u_{3}(0,0,1-r)=1$ (see figure 4 and (4.5)), there exists $\tau_{r} \in(1-r, r)$ such that

$$
\frac{\partial u_{3}}{\partial x_{3}}\left(0,0, \tau_{r}\right)=\frac{1}{1-2 r} .
$$

On the other hand, the Hopf maximum principle implies the positivity of the normal derivative of $u_{i}$ with respect to the plane $x_{3-i} O x_{3}$ for $i=1,2$ (see [5] for details), hence

$$
\frac{\partial u_{i}}{\partial x_{i}}(\hat{x})>0, \quad \text { for } i=1,2 .
$$

Let $\omega:=(-1,0) \times \mathbb{R}^{2} \backslash Q_{\#}$ and let $v_{1}$ be the function defined by

$$
v_{1}(x):=u_{1}(x)-\frac{x_{1}}{1-R}, \quad \text { for } x \in \omega .
$$

The function $v_{1}$ is harmonic in $\omega$ since $u_{1}$ is. Moreover, we have for any $x \in \partial \omega$,

- $v_{1}(x)=0$ if $x_{1}=0$,

- $v_{1}(x)=\frac{R}{1-R}>0$ if $x_{1}=-1$,

- for any connected component $L$ of $Q_{\#}$ such that $u_{1 \mid L}=-1, v_{1}(x)=-1-\frac{x_{1}}{1-R} \geq 0$ if $x \in L$, since the distance of $L$ to the plane $x_{1}=0$ is greater or equal to $1-R$ (see figure 4 ).

Hence, $v_{1} \geq 0$ on $\partial \omega$. Therefore, since $\left|v_{1}\right|$ is bounded from above by an affine function in the unbounded domain $\omega$, the maximum principle implies that $v_{1} \geq 0$ in $\omega$. As a consequence, since $u_{1}(\hat{x})=0$, we obtain for any $\tau \in(1-r, r)$,

$$
\frac{\partial u_{1}}{\partial x_{1}}(\hat{x})=\lim _{\substack{x_{1} \rightarrow 0 \\ x_{1}<0}}\left(\frac{u_{1}\left(x_{1}, 0, \tau\right)}{x_{1}}\right)=\lim _{\substack{x_{1} \rightarrow 0 \\ x_{1}<0}}\left(\frac{v_{1}\left(x_{1}, 0, \tau\right)}{x_{1}}+\frac{1}{1-R}\right) \leq \frac{1}{1-R} .
$$

A similar result holds for $i=2$, hence by (4.31) we get

$$
0<\frac{\partial u_{i}}{\partial x_{i}}(\hat{x}) \leq \frac{1}{1-R}, \quad \text { for } i=1,2
$$

This combined with (4.30) implies that, for any $r \in\left(\frac{1}{2}, \frac{3}{2}-R\right)$, the point $\hat{x}_{r}:=\left(0,0, \tau_{r}\right)$ satisfies the inequalities

$$
0<\frac{\partial u_{1}}{\partial x_{1}}\left(\hat{x}_{r}\right) \frac{\partial u_{2}}{\partial x_{2}}\left(\hat{x}_{r}\right)\left(\frac{\partial u_{1}}{\partial x_{1}}\left(\hat{x}_{r}\right)+\frac{\partial u_{2}}{\partial x_{2}}\left(\hat{x}_{r}\right)\right)^{-1} \leq \frac{1}{2(1-R)}<\frac{1}{2 r-1}=-\frac{\partial u_{3}}{\partial x_{3}}\left(\hat{x}_{r}\right),
$$


hence the limit (4.29) is negative for $\hat{x}_{r}$. Therefore, there exist $r, R, \kappa, \delta$ such that $s_{\delta}^{\kappa}<0$. For such values of $r, R, \kappa, \delta$, the homogenized $s$-coefficient $s_{*}$ defined by (4.23) is thus negative for small enough $\gamma$, which concludes the proof of Theorem 4.2.

Acknowledgements: The authors are very grateful to Dylon Whyte for permitting the reprinting of the Hitoye-Gusari mail (figure 1 and figure 2), and for suggesting the cubic chain mail as a generalization of the two-dimensional one we were first investigating. Marc Briane is grateful for support from the ACI-NIM plan lepoumonvousdisje grant 2003-45. Graeme Milton is grateful for support from the National Science Foundation through grant DMS-0411035, and from the Institut de Recherche Mathématique de Rennes (IRMAR) and the Université de Rennes 1 and thanks the Institut National des Sciences Appliquées de Rennes for its hospitality.

\section{References}

[1] A Ancona, "Some results and examples about the behavior of harmonic functions and Green's functions with respect to second order elliptic operators", Nagoya Math. J. 165 (2002), 123-158.

[2] P. Bauman, A. Marini \& V. Nesi, "Univalent solution of an elliptic system of partial differential equations arising in homogenization", Indiana Univ. Math. J., 50 (2) (2001), 747-757.

[3] D.J. Bergman, "Self duality and the low field Hall effect in 2D and 3D metal-insulator composites", in G. Deutscher, R. Zallen, and J. Adler eds., Percolation Structures and Processes 1983, 297-321.

[4] M. Briane, D. Manceau \& G.W. Milton, "Homogenization of the two-dimensional Hall effect", to appear.

[5] M. Briane, G.W. Milton \& V. Nesi, "Change of sign of the corrector's determinant for homogenization in three-dimensional conductivity", Arch. Rational Mech. Anal., 173 (2004), 133-150.

[6] F. Colombini \& S. Spagnolo, "Sur la convergence de solutions d'équations paraboliques", J. Math. Pures et Appl. 56 (1977), 263-306.

[7] B. Dacorogna, Direct Methods in the Calculus of Variations, in Applied Mathematical Sciences 78, Springer-Verlag Berlin Heidelberg 1989.

[8] R. LAKES, "Cellular solid structures with unbounded thermal expansion", J. Mater. Sci. Lett. 15 (1996) 475-477.

[9] L. Landau \& E. Lifchitz, Électrodynamique des Milieux Continus, Éditions Mir, Moscou 1969.

[10] N.G. Meyers, "An $L^{p}$-estimate for the gradient of solutions of second order elliptic divergence equations", Ann. Sc. Norm. Sup. Pisa, 17 (1963), 189-206.

[11] F. Murat \& L. TArtar, "H-convergence", Topics in the Mathematical Modelling of Composite Materials, L. Cherkaev and R.V. Kohn ed., Progress in Nonlinear Differential Equations and their Applications, Birkaüser, Boston (1998), 21-43.

[12] M. Ali Omar, Elementary Solid State Physics, Addison Wesley, Reading Massachusetts, World Student Series Edition 1975.

[13] O. Sigmund \& S. Torquato, "Composites with extreme thermal expansion coefficients", Appl. Phys. Lett., 69 (1996), 3203-3205.

[14] O. Sigmund \& S. Torquato, "Design of materials with extreme thermal expansion using a three-phase topology optimization method", J. Mech. Phys. Solids, 45 (1997), 1037-1067. 
[15] D. Stroud \& D.J. Bergman, "New exact results for the Hall-coefficient and magnetoresistance of inhomogeneous two-dimensional metals", Physical Review B (Solid State), 30 (1984), 447-449.

[16] L. TARTAR, "Compensated compactness and applications to partial differential equations", Nonlinear Analysis and Mechanics, Research Notes in Mathematics, ed. by R.J. Knops, Vol. 39, Pitman 1979, 136-212.

[17] "Levi-Civita symbol", Wikipedia, the free encyclopedia, http://en.wikipedia.org/wiki/Levi-Civita_symbol.

\section{A Proof of part (ii) of Theorem 2.7}

The corrector $P^{\varepsilon}(h)$, for $h \in O$, of Proposition 2.5 associated with the matrix-valued function $A^{\varepsilon}(h)$, reads as

$$
P^{\varepsilon}(h)=I+D\left(\left[\operatorname{Div}\left(A^{\varepsilon}(h) D^{\cdot}\right)\right]^{-1}\left[\operatorname{Div}\left(A^{*}(h)-A^{\varepsilon}(h)\right)\right]\right),
$$

where $\operatorname{Div}\left(A^{\varepsilon}(h) D^{\cdot}\right)$ is an invertible operator from $H_{0}^{1}(\Omega)^{d}$ onto $H^{-1}(\Omega)^{d}$. Hence, since $A^{\varepsilon}(h)$ and $A^{*}(h)$ admit a first-order expansion in the neighbourhood of $h=0$ and the operator $\operatorname{Div}\left(A^{\varepsilon}(0) D^{*}\right)$ is invertible, $P^{\varepsilon}(h)$ also admits the following first-order expansion

$$
P^{\varepsilon}(h)=P^{\varepsilon}+\mathcal{P}_{1}^{\varepsilon} \cdot h+o_{L^{2}(\Omega) d \times d}(h),
$$

where $P^{\varepsilon}=P^{\varepsilon}(0)$ is the corrector of Proposition 2.5 associated with $A^{\varepsilon}=A^{\varepsilon}(0)$. As $\mathcal{P}_{1}^{\varepsilon}$ is the first derivative of $P^{\varepsilon}(h)$ at $h=0, \mathcal{P}_{1}^{\varepsilon} \cdot h$ is also a gradient for any $h \in O$. Moreover, since $P^{\varepsilon}(h)$ and $P^{\varepsilon}$ weakly converge to $I$ in $L^{2}(\Omega)^{d \times d}$ as $\varepsilon \rightarrow 0$, the expansion (A.2) combined with the lower semicontinuity of the $L^{2}$-norm implies that $\mathcal{P}_{1}^{\varepsilon}$ weakly converges (up to a subsequence) to some $\mathcal{P}_{1}$ in $L^{2}(\Omega)^{d \times d \times d}$, such that $\mathcal{P}_{1} \cdot h=o_{L^{2}(\Omega) d \times d}(h)$, hence $\mathcal{P}_{1}=0$. By uniqueness of the limit the whole sequence $\mathcal{P}_{1}^{\varepsilon}$ weakly converges to 0 .

On the other hand, the first-order expansions (2.6) and (A.2) yield

$$
\begin{aligned}
& P^{\varepsilon}(h)^{T} A^{\varepsilon}(h) P^{\varepsilon}(h) \\
& =\left(P^{\varepsilon}+\mathcal{P}_{1}^{\varepsilon} \cdot h\right)^{T}\left(A^{\varepsilon}+\mathcal{A}_{1}^{\varepsilon} \cdot h\right)\left(P^{\varepsilon}+\mathcal{P}_{1}^{\varepsilon} \cdot h\right)+o_{L^{1}(\Omega) d \times d}(h) \\
& =\left(P^{\varepsilon}\right)^{T} A^{\varepsilon} P^{\varepsilon}+\left(P^{\varepsilon}\right)^{T}\left(\mathcal{A}_{1}^{\varepsilon} \cdot h\right) P^{\varepsilon}+\left(P^{\varepsilon}\right)^{T} A^{\varepsilon}\left(\mathcal{P}_{1}^{\varepsilon} \cdot h\right)+\left(\mathcal{P}_{1}^{\varepsilon} \cdot h\right)^{T} A^{\varepsilon} P^{\varepsilon}+o_{L^{1}(\Omega)^{d \times d}}(h) \\
& =\left(P^{\varepsilon}\right)^{T} A^{\varepsilon} P^{\varepsilon}+\left(P^{\varepsilon}\right)^{T}\left(\mathcal{A}_{1}^{\varepsilon} \cdot h\right) P^{\varepsilon}+\left(A^{\varepsilon} P^{\varepsilon}\right)^{T}\left(\mathcal{P}_{1}^{\varepsilon} \cdot h\right)+\left(\mathcal{P}_{1}^{\varepsilon} \cdot h\right)^{T} A^{\varepsilon} P^{\varepsilon}+o_{L^{1}(\Omega)^{d \times d}}(h),
\end{aligned}
$$

by using the symmetry of $A^{\varepsilon}$. Since $A^{\varepsilon} P^{\varepsilon}$ has a compact divergence in $H^{-1}(\Omega)^{d}$ and $P^{\varepsilon}(h), P^{\varepsilon}, \mathcal{P}_{1}^{\varepsilon} \cdot h$ are sequences of gradients which weakly converge in $L^{2}(\Omega)^{d \times d}$ respectively to $I, I$, 0 , the div-curl lemma implies that

$$
\left(P^{\varepsilon}\right)^{T}\left(\mathcal{A}_{1}^{\varepsilon} \cdot h\right) P^{\varepsilon}+o_{L^{1}(\Omega) d \times d}(h) \longrightarrow A^{*}(h)-A^{*} \quad \text { weakly-* in } \mathcal{M}(\Omega)^{d \times d},
$$

where $\mathcal{M}(\Omega)$ denotes the space of the Radon measures on $\Omega$. Therefore, thanks to the boundedness of $\left(P^{\varepsilon}\right)^{T}\left(\mathcal{A}_{1}^{\varepsilon} \cdot h\right) P^{\varepsilon}$ in $L^{1}(\Omega)^{d \times d}$, combined with the linearity of $\mathcal{A}_{1}^{\varepsilon}$, there exists a subsequence $\varepsilon^{\prime}$ such that, for any $h \in O$ and any $t \in \mathbb{R}$,

$$
A^{*}(t h)=A^{*}+t \lim _{\mathcal{M}(\Omega)^{d \times d}}\left(P^{\varepsilon^{\prime}}\right)^{T}\left(\mathcal{A}_{1}^{\varepsilon^{\prime}} \cdot h\right) P^{\varepsilon^{\prime}}+o_{L^{1}(\Omega)} d \times d(t),
$$

where $\lim _{\mathcal{M}(\Omega) d \times d}$ denotes the limit as $\varepsilon^{\prime} \rightarrow 0$ in the weak-* sense of the Radon measures. Equating the above expression with (2.7) in which $h$ is replaced by $t h$, and dividing the resulting equality by $t \neq 0$, then passing to the limit as $t \rightarrow 0$, we get

$$
\lim _{\mathcal{M}(\Omega)^{d \times d}}\left(P^{\varepsilon^{\prime}}\right)^{T}\left(\mathcal{A}_{1}^{\varepsilon^{\prime}} \cdot h\right) P^{\varepsilon^{\prime}}=\mathcal{A}_{1}^{*} \cdot h .
$$

Using a uniqueness argument the former limit holds actually for the whole sequence $\varepsilon$, which yields the desired convergence (2.8). 